\title{
Transcriptome analysis reveals the mechanism of internode development affecting maize stalk strength
}

\author{
Liuyong Xie ${ }^{\dagger}$, Daxing Wen ${ }^{\dagger}$, Chenglai Wu and Chunqing Zhang ${ }^{*}$
}

\begin{abstract}
Background: The stalk rind is one of the important factors affecting maize stalk strength that is closely related to stalk lodging. However, the mechanism of rind development in maize is still largely unknown.

Results: In this study, we analyzed the mechanical, anatomical, and biochemical properties of the third basal internode in one maize non-stiff-stalk (NSS) line and two stiff-stalk (SS) lines. Compared with the NSS line, the two SS lines had a significantly higher rind penetrometer resistance, thicker rind, and higher dry matter, hemicellulose, cellulose, and lignin weights per unit length. RNA-seq analysis was used to compare transcriptomes of the third basal internode of the two SS lines and the NSS line at the ninth leaf and tasseling stages. Gene Ontology (GO) enrichment analysis revealed that genes involved in hydrolase activity (hydrolyzing O-glycosyl compounds) and cytoskeleton organization were significantly up-regulated in the two SS lines at the ninth leaf stage and that microtubule process-related genes were significantly up-regulated in the two SS lines at the tasseling stage. Moreover, the two SS lines had enhanced expression of cell wall metabolism-related genes at the tasseling stage.
\end{abstract}

Conclusions: The synthesis of cell wall polysaccharides and the cytoskeleton might play important roles in internode development. Our results can be applied for screening lodging-resistant inbred lines and breeding lodging-resistant cultivars in maize.

Keywords: Maize, Lodging, Stalk strength, Rind, Cell wall

\section{Background}

Stalk lodging affects not only maize yield but also mechanized harvesting efficiency. During lodging, the spatial distribution of leaves is disturbed, which alters photosynthetic efficiency. Stalk breakage may hinder sufficient transport of water and nutrients or lead to death of the entire plant. In addition, maize stalk lodging reduces ear position, which affects mechanized harvesting efficiency. Previous studies have shown that strong stalks significantly reduce stalk lodging $[1,2]$. Therefore, studying

\footnotetext{
*Correspondence: cqzhangsdau@163.com

'Liuyong Xie and Daxing Wen contributed equally to this work.

State Key Laboratory of Crop Biology, Agronomy College, Shandong

Agricultural University, Tai'an, Shandong Province 271018, P. R. China
}

mechanisms regulating stalk strength may provide new insights into stalk lodging resistance.

The maize stalk is composed of the pith and the rind, the latter mainly consisting of the epidermis and subepidermal lignified sclerenchyma cells [3]. Sclerenchyma cells have a thickened secondary cell wall that affects the strength and rigidity of plant tissues [4]. Therefore, rind thickness is closely related to rind penetrometer resistance (RPR) [5]. Optimization of cell wall synthesis and assembly can increase cell wall thickness, which is critical for improving stalk strength. However, the mechanism of sclerenchyma development in maize stalks is still unclear.

The primary cell wall, consisting of polysaccharides and smaller amounts of structural proteins, is synthesized in growing cells [6]. Once growth ceases, sclerenchyma cells 
deposit a thick lignified secondary wall containing more polysaccharides. Cellulose consisting of $\beta$-1,4-linked glucan chains is the main polysaccharide of plant cell walls [7]. In addition, many matrix polysaccharides, mainly pectin and hemicellulose, have been found in plant cell walls. Pectin polysaccharides include homogalacturonan, and rhamnogalacturonan I and II [8]. Hemicelluloses in plant cell walls include mannans, glucomannans, xylans, xyloglucans, and $\beta-(1 \rightarrow 3,1 \rightarrow 4)$-glucans [9]. Cellulose is synthesized by cellulose synthase (CesA) complexes (CSCs) embedded in the plasma membrane and is organized as microfibrils along the direction of microtubules. Disruption of cortical microtubules, which has revealed that membrane-localized CSCs are disorganized, does not affect the transport of CSCs from endomembranes to the plasma membrane or the velocity of CSCs at the plasma membrane [10]. Non-cellulosic polysaccharides are assembled in the Golgi apparatus and transported to the cell wall via vesicles [11]. When the cytoskeleton is damaged, vesicular transport and deposition at the cell walls are affected [12]. Improvement of maize stalk strength requires clarification of the stalk cell wall synthesis pathways. Although some studies on cell wall synthesis have been carried out, the internode development of maize inbred lines having different stalk strengths at different developmental stages remains poorly understood.

In this study, we analyzed the mechanical, anatomical, and biochemical properties of the third basal internode in one NSS line and two SS lines. We found that the two SS lines had a higher RPR than the NSS line. Further analysis revealed that the two SS lines had thicker rinds, rind and vascular bundle sheath cell walls, and more dry matter, hemicellulose, cellulose, and lignin per unit length than the NSS line. To explore genes and gene networks potentially playing important roles in regulating stalk strength in maize, we analyzed the transcriptomes of the third basal internode in the three inbred lines having different stalk strengths at the ninth leaf and tasseling stages. According to our results, genes involved in hydrolase activity (hydrolyzing O-glycosyl compounds) and cytoskeleton organization were significantly up-regulated in the two SS lines at the ninth leaf stage. Moreover, the two SS lines exhibited enhanced expression of cell wall metabolism-related genes at the tasseling stage. Taken together, our results provide new insights into the mechanism of internode development affecting maize stalk strength.

\section{Results}

\section{Comparison of stalk strength-related traits among three} maize inbred lines

To identify differences in stalk strength, we analyzed RPR in one NSS and two SS inbred lines. No significant difference in RPR was detected between the two SS lines and the NSS line at the ninth leaf stage, but the two SS lines had markedly higher RPRs than the NSS line at the tasseling and maturity stages (Fig. 1A-B). Although SS2 was taller and had notably longer stem diameters and internode lengths than NSS, no significant difference was observed in the three traits between NSS and SS1 (Fig. S1). To investigate the effects of cell wall structural material contents on stalk strength, we measured the dry weight per unit length (DWUL), hemicellulose (HWUL), cellulose (CWUL), and lignin (LWUL) weights per unit length of the third basal internode (Fig. 1C-F). No significant difference was found in DWUL, CWUL, and LWUL between the two SS lines and the NSS line at the ninth leaf stage, but HWUL in the NSS line was higher than those in the two SS lines. At the tasseling and maturity stages, the two SS lines had higher DWUL and cell wall structural material contents (HWUL, CWUL, and LWUL) than the NSS line. The DWUL and cell wall structural material contents (HWUL, CWUL, and LWUL) of the two SS lines gradually increased from the ninth leaf stage to the maturity stage. In the NSS line, LWUL exhibited a similar trend as in the two SS lines, whereas HWUL decreased over time. Moreover, DWUL and CWUL in the NSS line first increased and then decreased from the ninth leaf stage to the maturity stage.

To investigate differences in the stalk microstructure of the NSS line and the two SS lines, we observed cross sections of the third basal internode at the ninth leaf and tasseling stages (Fig. 2A). No significant difference in stalk microstructure was detected between the NSS line and the two SS lines at the ninth leaf stage. At the tasseling stage, no significant difference was observed in the pith of the stem between the NSS line and the two SS lines. However, the two SS lines had notably thicker rinds than the NSS line (Fig. 2A). We subsequently measured stalk rind, rind cell wall, vascular bundle sheath (VBS), and VBS cell wall thicknesses at the tasseling stage (Fig. 2B-E). The rind, VBS, rind cell, and VBS cell wall thicknesses of the two SS lines were markedly larger than those of the NSS line at the tasseling stage. Although there was no significant difference in VBS thickness between the SS1 line and NSS line, the SS2 line had a notably larger VBS thickness than the NSS line. At the ninth leaf stage, the early stage of stalk structure formation, rind tissue development was incomplete. At the tasseling stage, the two SS lines had significantly thicker rind and thick-walled cells than the NSS line. Therefore, the differences in stalk strength between the two SS lines and the NSS line were associated with rind and cell wall thicknesses. 

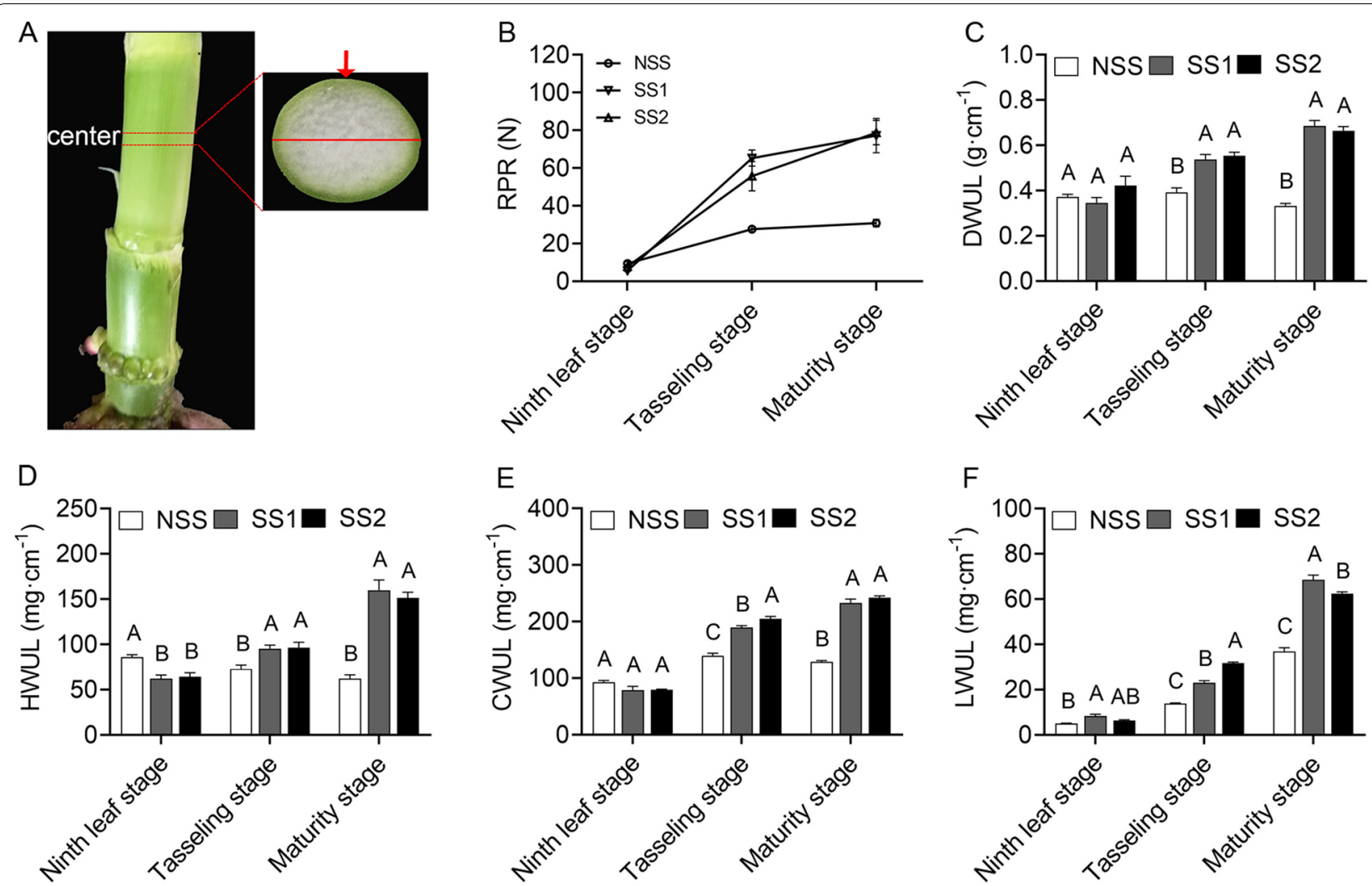

Fig. 1 Rind penetrometer resistance (RPR) and stalk chemistry composition of two stiff-stalk (SS) lines and a non-stiff-stalk (NSS) line. A Stem region subjected to RPR measurement, paraffin sectioning, and RNA-seq sampling. The cross-sectional area of the probe was $1 \mathrm{~mm}^{2}$. Approximately $2 \mathrm{~mm}$ of the stem center was separated along the long axis of the cross section. The red arrow indicates the location of the RPR measurement, and the red line indicates the long axis. B RPR of the third basal internode. C-F Dry weight per unit length (DWUL), hemicellulose content per unit length (HWUL), cellulose content per unit length (CWUL), and lignin content per unit length (LWUL) of the third basal internode. Error bars represent standard deviation $(n=3)$. Different letters indicate significant differences at the same developmental stage ( $p<0.01$ by one-way ANOVA)

\section{Comparison of the expression levels of genes related to cell wall metabolism between the two SS lines and the NSS line}

To explore the mechanism by which internode development affects maize stalk strength, we analyzed the transcriptomes of the third basal internode at two stages (ninth leaf stage and tasseling stage) by RNA-seq. After removing low-quality regions and adapter sequences, approximately 51.16 to 76.11 million clean reads remained (Table S1). Approximately 46.12 to 68.81 million clean reads were mapped to the maize B73 reference genome (v4.0). In each library, 80.09 to $88.59 \%$ of clean reads were uniquely mapped reads (Table $\mathrm{S} 1$ ). In a principal component analysis (PCA), biological replicates of the same treatment clustered together, which implied that the transcriptome datasets were satisfactory (Fig. 3A). The first principal component was able to discriminate between samples at the ninth leaf stage and the tasseling stage, which indicates that the transcriptome datasets from these two stages were markedly different.
Genes with fragments per kilobase of transcript sequence per million base pairs sequenced (FPKM) $\geq 1$ were considered to be expressed genes. According to this criterion, 21,819, 21,663, and 22,023 genes were expressed in NSS, SS1, and SS2, respectively, at the ninth leaf stage. At the tasseling stage, there were 20,882, 21,241 , and 20,984 expressed genes in NSS, SS1, and SS2, respectively. After filtering out genes with low expression (FPKM $<1$ in all three inbred lines), 25,751 and 25,088 genes were expressed at the ninth leaf and tasseling stages, respectively. We then used the $R$ package DESeq to identify differentially expressed genes (DEGs). Compared with the NSS line, approximately 8000 DEGs each were significantly up-regulated and significantly down-regulated in the two SS lines (Fig. 3B). To reduce the effects of material background on the results, we applied a Venn diagram to screen common DEGs in SS1vsNSS and SS2vsNSS (Fig. 3C-D). With regard to upregulated DEGs, we identified 3973 and 4338 common DEGs at the ninth leaf and tasseling stages, respectively. 


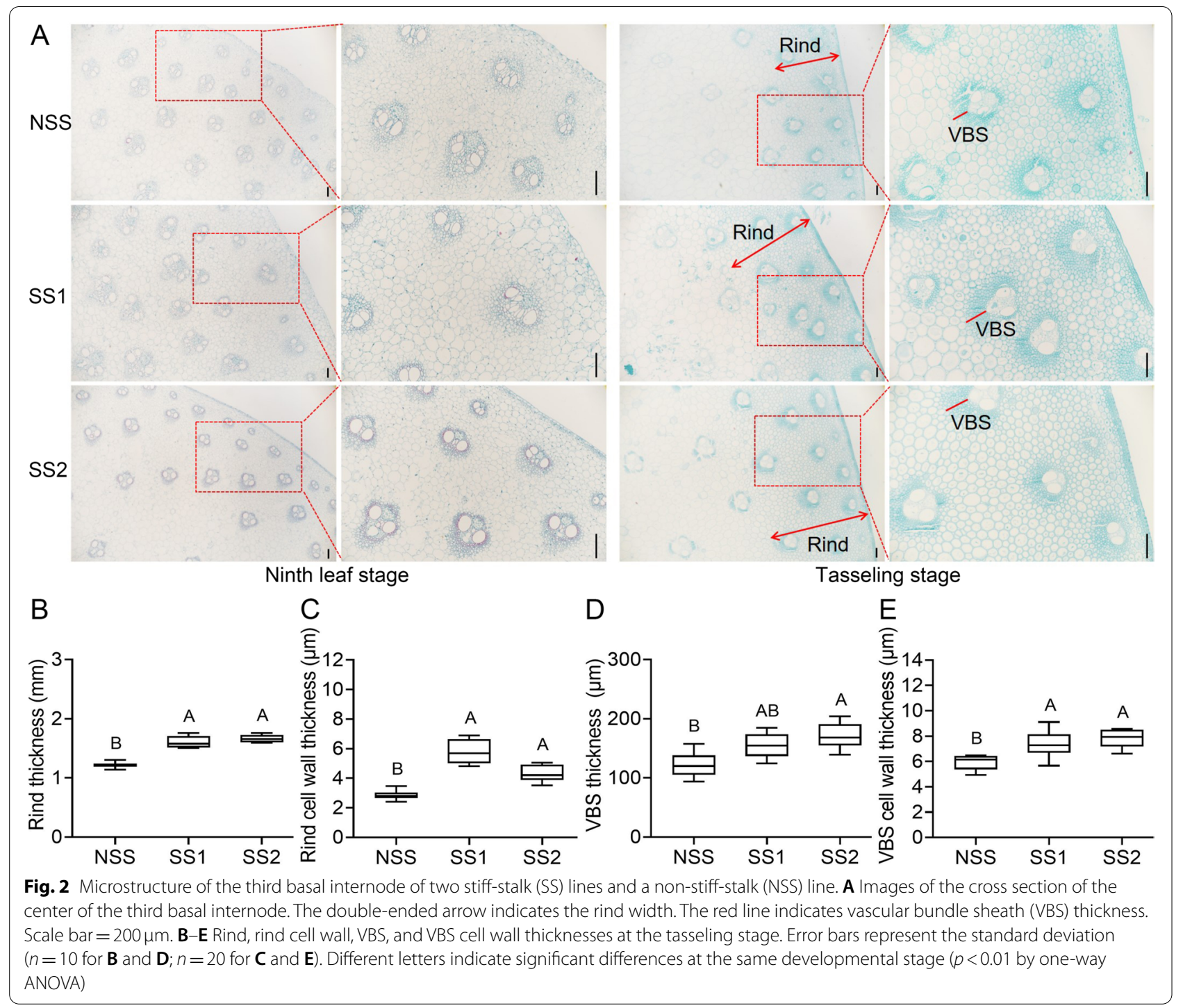

To further analyze the function of these common DEGs, we performed a Gene Ontology (GO) enrichment analysis $(p<0.05)$. In a subsequent transcriptome analysis, we focused on DEGs and GO terms involved in cell wall metabolism given the differences in thick-walled cells between the two SS lines and the NSS line.

\section{Involvement of hydrolyzing 0-glycosyl compounds and cytoskeleton organization-related genes in internode development at the ninth leaf stage}

There were 3973 common up-regulated DEGs in SS1vsNSS and SS2vsNSS at the ninth leaf stage (Fig. 3C). GO enrichment analysis of these common up-regulated DEGs revealed that the most significantly enriched GO terms were photosynthesis (GO: 0015979, $\left.p=8.79 \times 10^{-6}\right)$ in the biological process group, thylakoid (GO: 0009579, $p=1.24 \times 10^{-5}$ ) in the cellular component group, and hydrolase activity (hydrolyzing O-glycosyl compounds) (GO: 0004553, $p=6.19 \times 10^{-4}$ ) in the molecular function group (Fig. 4). Among them, 64 DEGs were involved in hydrolase activity (hydrolyzing O-glycosyl compounds) (GO: 0004553, $p=6.19 \times 10^{-4}$, Table S2). Many of these 64 DEGs were involved in cell wall metabolism. The largest group comprised glycosyl hydrolase family genes (36 DEGs). In addition, genes related to xyloglucan endo-transglycosylase (XET, Zm00001d002410, Zm00001d014613, Zm00001d014617, $Z m 00001 d 017699$, and Zm00001d029814), beta-glucosidase 2 (Zm00001d005431 and Zm00001d048988), and cellulase $(Z m 00001 d 010039)$ were markedly up-regulated in SS1vsNSS and SS2vsNSS. Moreover, 11 genes implicated in cytoskeleton organization (GO: 0007010, $\left.p=1.00 \times 10^{-2}\right)$ (Table S3) were notably up-regulated in the SS lines. Therefore, the two SS lines had significantly 


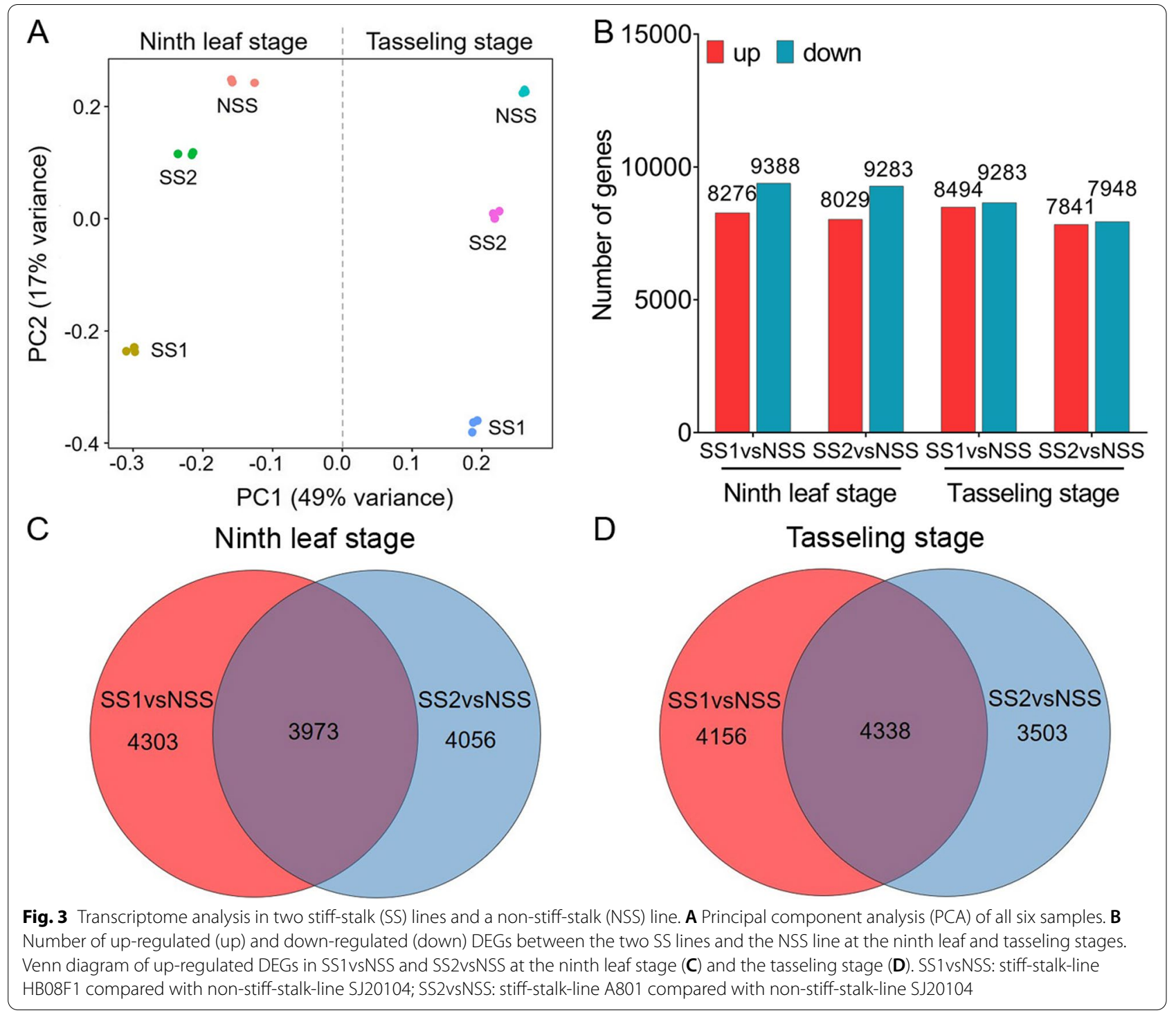

higher expression levels of cell wall metabolism-related genes than the NSS line, which suggests that the difference in cell wall metabolism between the NSS line and the two SS lines had already occurred at the ninth leaf stage.

\section{Cell wall metabolism-related genes are implicated} in the internode development of inbred lines with different stalk strengths at the tasseling stage

At the tasseling stage, 4338 DEGs were commonly upregulated in SS1vsNSS and SS2vsNSS (Fig. 3D). For these commonly up-regulated DEGs, the most remarkably significantly enriched GO terms were the microtubule-based process (GO: 0007017, $p=1.52 \times 10^{-5}$ ) in the biological process group, chromosomal part (GO: 0044427, $p=2.36 \times 10^{-4}$ ) in the cellular component group, and protein heterodimerization activity (GO: 0046982, $p=1.06 \times 10^{-8}$ ) in the molecular function group (Fig. 5). In regard to microtubule-based processes (GO: 0007017, $\left.p=1.52 \times 10^{-5}\right)$, more than half $(20 / 36)$ of the DEGs encoded proteins that were predicted to have a kinesin motor domain (Table S4). In Arabidopsis thaliana, kinesin-4-based transport of non-cellulose substances along cortical microtubules is associated with cell wall mechanics [13]. Therefore, we speculate that DEGs involved in microtubule-based processes might affect the supply of synthetic substances in the cell wall. Moreover, many GO terms involved in cell wall metabolism were significantly enriched in the biological process group, 


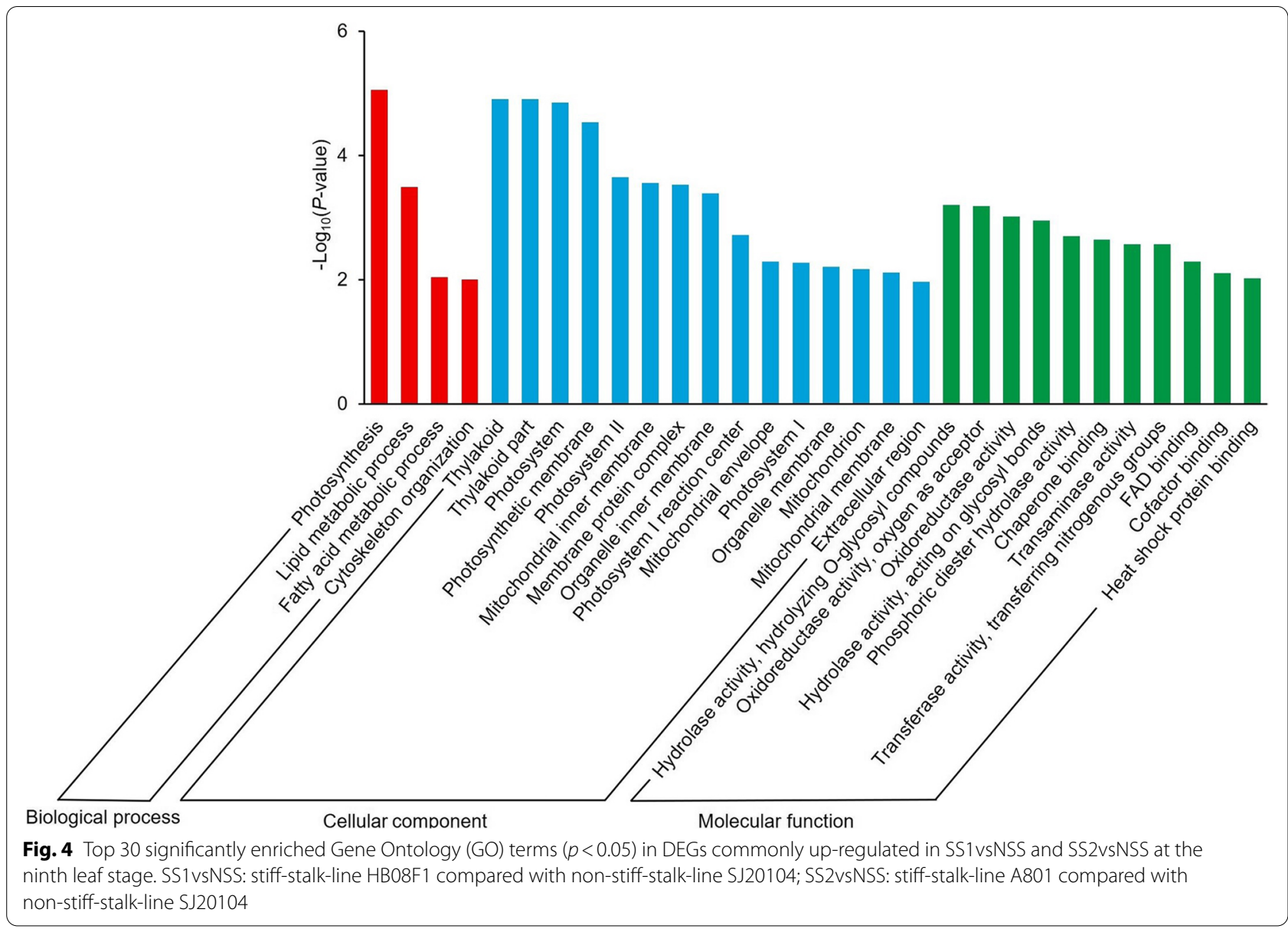

such as plant-type cell wall biogenesis (GO: 0009832, $p=4.76 \times 10^{-4}$ ), cellulose microfibril organization (GO: 0010215, $p=4.76 \times 10^{-4}$ ), and cell wall assembly (GO: 0070726, $p=4.76 \times 10^{-4}$ ). Many more cell wall metabolism-related GO terms were enriched at the tasseling stage than at the ninth leaf stage, which indicates that differences in cell wall metabolism between the NSS line and the two SS lines became more pronounced at the tasseling stage.

Many genes related to the synthesis of cell wall components were significantly enriched in transferase activity (transferring hexosyl groups) (GO: 0016758, $\left.p=6.46 \times 10^{-5}\right)$. Most of the transferase activity related genes were UDP-glucoronosyl and UDP-glucosyl transferase genes (33/85, Table S5). The glycosyltransferase (GT) family GT43 is involved in the biosynthesis of xylan, which is a hemicellulose $[14,15]$. Compared with the NSS line, 12 GT43 family genes were up-regulated in the two SS lines at the tasseling stage (Fig. 6). In addition, although the two SS lines had higher CWUL than the NSS line at the tasseling stage, 11 cellulose synthase genes were still significantly up-regulated in the two SS lines compared with the NSS line, thereby resulting in further enlargement of the difference in CWUL between the NSS line and the two SS lines at the maturity stage (Figs. 1E and 6 and Table S5). Lignin synthesis-related GO terms were not notably enriched at the tasseling stage, but the two SS lines already had significantly higher LWUL than the NSS line. Therefore, we also analyzed the expression pattern of lignin synthesis-related genes, including phenylalanine ammonialyase $(P A L)$, 4-coumarate:CoA ligase $(4 C L)$, and laccase. $P A L$ and $4 C L$ are involved in lignin synthesis [16, 17], and laccase genes involved in lignin polymerization [18] have been reported in Arabidopsis and maize. The results showed that five $P A L$ genes, five $4 C L$ genes, and six laccase genes were up-regulated in both SS lines (Fig. 6 and Table S6). These genes might play important roles in the biosynthesis of cell wall components and enhancing stalk strength.

Hemicellulose and cellulose deposition form the secondary cell wall network, which affects cell wall mechanics [19, 20]. Xyloglucan endotransglucosylase (XET) cuts and rejoins hemicellulose chains to promote plant cell wall assembly and growth 


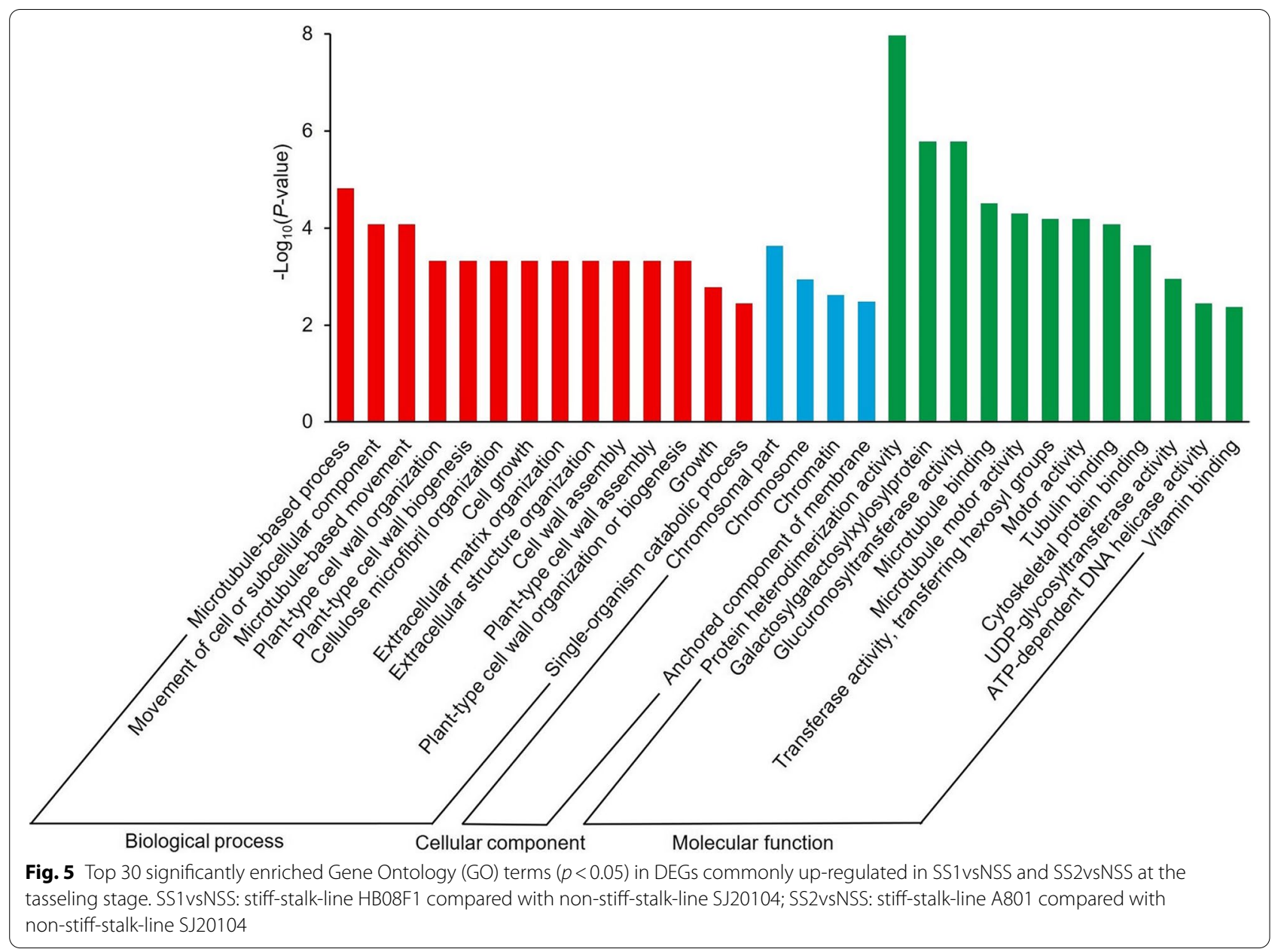

regulation [21]. Previous studies have identified a role for XET in secondary cell wall deposition [22]. Three XET (Zm00001d024386, Zm00001d024071, and $Z m 00001 d 002412)$ genes were up-regulated in the two SS lines at the tasseling stage (Fig. 6 and Table S5). Mutation in the gene encoding a COBRA-like protein in Arabidopsis affects cellulose deposition [23]. We found that six DEGs were enriched in plant-type cell wall organization (GO: 0009664, $p=4.76 \times 10^{-4}$ ). All six DEGs were predicted to encode COBRA-like proteins and were up-regulated in the two SS lines at the tasseling stage (Fig. 6 and Table S7). In rice, the deletion of a COBRA-like protein gene causes a reduction in cellulose content, resulting in decreases in secondary cell wall thickness and mechanical strength [24]. In particular, mutants of brittle stalk2 (Zm00001d047276) display brittle organs (including stems) [25]. Therefore, many genes involved in cell wall metabolism were upregulated in the two SS lines compared with the NSS line, which is consistent with the observed trends in cell wall structural material contents.

\section{GO term enrichment analysis of down-regulated DEGs in SS1vsNSS and SS2vsNSS}

At the ninth leaf stage, 5315 common DEGs were down-regulated in SS1vsNSS and SS2vsNSS (Fig. S2A). Among these common down-regulated DEGs, GO term enrichment analysis showed that the most significantly enriched GO terms were sulfate transport (GO: 0008272, $p=5.13 \times 10^{-4}$ ) and sulfur compound transport (GO: 0072348, $p=5.13 \times 10^{-4}$ ) in the biological process group and sulfate transmembrane transporter activity (GO: 0015116, $p=4.69 \times 10^{-4}$ ) in the molecular function group (Fig. S3). There was no significant GO term that might be involved in stalk strength. Venn diagram analysis revealed that 5138 common DEGs were downregulated in SS1vsNSS and SS2vsNSS at the tasseling stage (Fig. S2B). The most significantly enriched GO terms related to these common down-regulated DEGs 


\begin{tabular}{|c|c|c|c|c|c|c|c|c|c|c|}
\hline & & & & & Cellulose synthesi & & & Lignin synthesis & & \\
\hline 0 & 2 & 4 & 6 & 10 & Cellulose synthas & $(\operatorname{Ces} A)$ & & Phenylalnine amm & onialyase $(P$ & $A L)$ \\
\hline & & $\log _{2}$ & (Fold change) & & & SS1VsNSS & SS2vsNSS & & SS1vsNSS & SS2vsNSS \\
\hline & & & & & Zm00001d032776 & & & Zm00001d017276 & & \\
\hline $\mathrm{Hem}$ & ulos & syn & thesis & & Zm00001d043477 & & & $Z m 00001 d 051166$ & & \\
\hline Glyce & tran & feras & e family 43 & GT43) & $Z m 00001 d 005451$ & & & $Z m 00001 d 017279$ & & \\
\hline & & & SS1vsNSS & SS2vsNSS & Zm00001d005775 & & & Zm00001d051163 & & \\
\hline Zmoo & do4 & & & & $Z m 00001 d 020531$ & & & Zm00001d017274 & & \\
\hline Zmoo & do2 & & & & Zm00001d019149 & & & & & \\
\hline $\mathrm{ZmoO}$ & d 01 & & & & $Z m 00001 d 005250$ & & & 4-coumarate:CoA & igase (4CL) & \\
\hline$Z m o 0$ & $d 01$ & & & & Zm00001d019507 & & & & SS1vsNSS & SS2vsNSS \\
\hline $\mathrm{ZmoO}$ & $d 04$ & & & & $Z m 00001 d 034553$ & & & Zm00001d015025 & & \\
\hline Zmoo & $d 03$ & & & & Zm00001d005478 & & & Zm00001d050224 & & \\
\hline Zmoo & $d 01$ & & & & Zm00001d045557 & & & $Z m 00001 d 026649$ & & \\
\hline $\mathrm{ZmoO}$ & do3 & & & & & & & Zm00001d001849 & & \\
\hline Zmoo & $d 02$ & & & & & & & Zm00001d027519 & & \\
\hline Zmoo & $d 00$ & & & & & & & & & \\
\hline Zmoo & $d 00$ & & & & Cellulose depositic & & & Lignin polymerizati & & \\
\hline Zmoo & $d 02$ & & & & COBRA-like prote & & & Laccase & & \\
\hline & & & & & & SS1vsNSS & SS2vsNSS & & SS1vsNSS & SS2vsNSS \\
\hline Hemi & lulos & dep & osition & & $Z m 00001 d 022081$ & & & Zm00001d042905 & & \\
\hline Xylog & $\operatorname{san} \epsilon$ & do-t & ransglycosyla & ase (XET) & Zm00001d022082 & & & Zm00001d042901 & & \\
\hline & & & SS1vsNSS & SS2vsNSS & Zm00001d047276 & & & $Z m 00001 d 042906$ & & \\
\hline $\mathrm{ZmoO}$ & 102 & & & & Zm00001d034049 & & & $Z m 00001 d 012408$ & & \\
\hline Zmoo & 100 & & & & $Z m 00001 d 013256$ & & & Zm00001d038371 & & \\
\hline Zmoo & $1 d 02$ & & & & Zm00001d007208 & & & $Z m 00001 d 028599$ & & \\
\hline
\end{tabular}

Fig. 6 Heatmap of genes related to cell wall synthesis at the tasseling stage. SS1vsNSS: stiff-stalk-line HB08F1 compared with non-stiff-stalk-line SJ20104; SS2vsNSS: stiff-stalk-line A801 compared with non-stiff-stalk-line SJ20104

were photosynthesis (GO: 0015979, $p=9.65 \times 10^{-11}$ ) under biological process, the thylakoid (GO: 0009579, $p=3.18 \times 10^{-12}$ ) and thylakoid part (GO: 0044436, $p=3.18 \times 10^{-12}$ ) under cellular components, and ironsulfur cluster binding (GO: 0051536, $p=1.80 \times 10^{-6}$ ) and metal cluster binding (GO: 0051536, $p=1.80 \times 10^{-6}$ ) under molecular function (Fig. S4). GO terms that might be involved in stalk strength were also not found at the tasseling stage. Therefore, up-regulated DEGs between the two SS lines and the NSS line might play more important roles in regulating stalk strength than down-regulated DEGs.

\section{Identification of differentially expressed transcription factors}

Transcription factors (TFs) play important roles in cell wall metabolism [20]. To explore which TFs might be involved in internode development, we identified common differentially expressed TFs between SS and NSS lines by annotation in the PlantTFDB (http://planttfdb. gao-lab.org/index.php?sp=Zma) database. There were 514 differentially expressed TFs assigned to 45 families at the ninth leaf stage. The top 10 families by TF number were AP2/ERF, MYB, bHLH, bZIP, WRKY, NAC, C2H2, HD-ZIP, G2-like, and GRAS (Fig. 7A, Table S8). At the tasseling stage, 521 differentially expressed TFs were assigned to 47 families. The top 10 families by TF number were bHLH, AP2/ERF, bZIP, MYB, NAC, C2H2, GRAS, WRKY, HD-ZIP, and MYB_related (Fig. 7B, Table S8). AP2/ERF family TFs can regulate secondary cell wall formation [26]. In the present study, the AP2/ERF, bHLH, MYB, and bZIP families had notably more DEGs than the other families at both the ninth leaf and tasseling stages. The MYB and NAC families play important roles in the network of TFs that regulate secondary wall synthesis [27]. At the tasseling stage, NAC families also had a large number of differentially expressed TFs. These differentially expressed TFs might participate the in regulation of maize stalk strength, which needs validation in further study.

\section{Validation of RNA-seq data by qRT-PCR}

To validate the DEGs identified by RNA-seq, we performed qRT-PCR assays using independently collected samples identical to those used for RNA-seq analysis at the same developmental stage. Among the six DEGs selected for validation, three DEGs were up-regulated between the two SS lines and the NSS line at the ninth leaf and tasseling stages (Fig. 8). Zm00001d039004 encodes a NAC-transcription factor that shares high protein sequence homology with the secondary wallassociated NAC domain 2 (SND2) protein in rice [28]. 


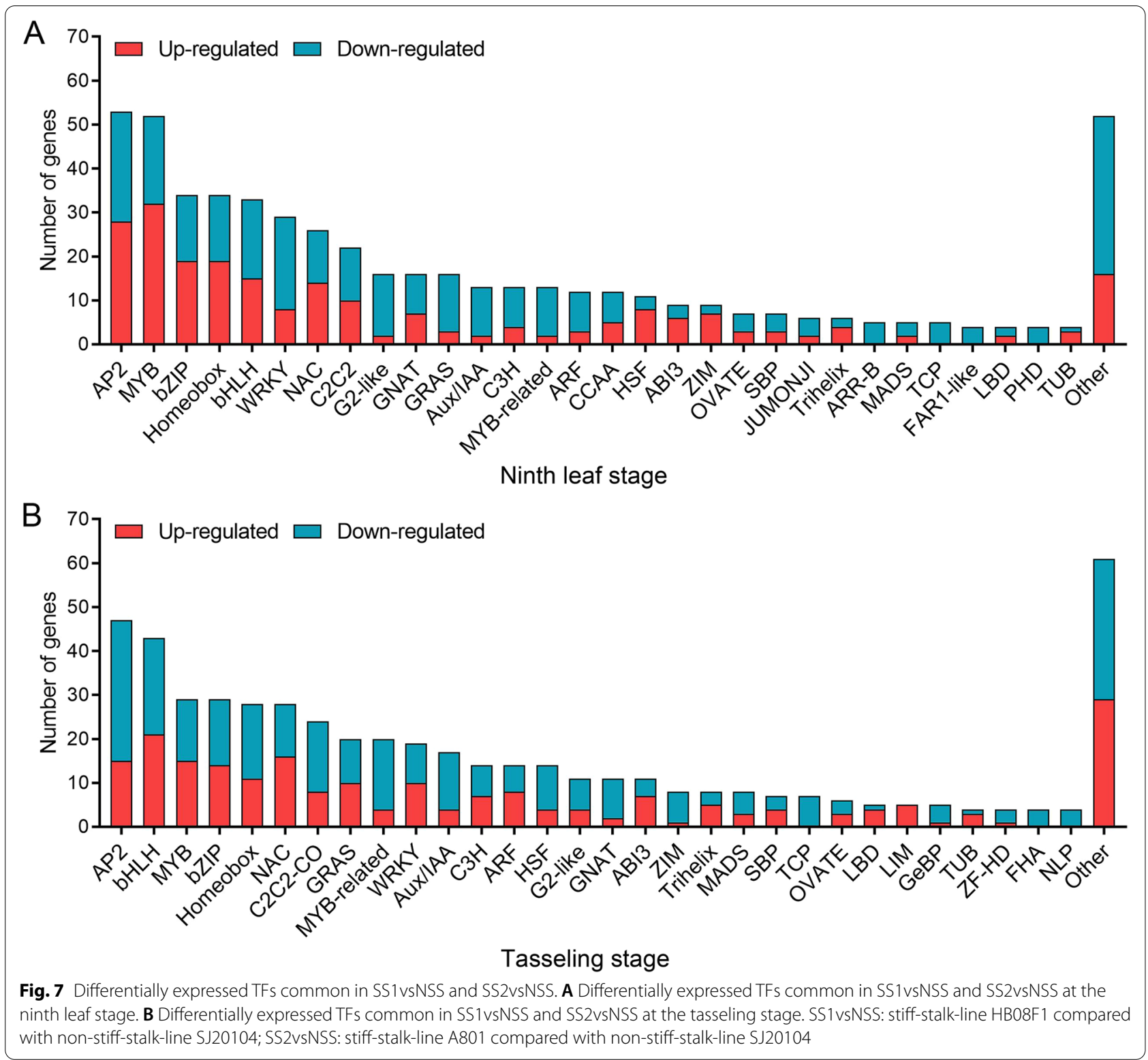

$Z m 00001 d 029343$ was predicted to encode an F-actin capping protein. The Zm00001d047453 encoded protein was predicted to have a villin headpiece domain. At the tasseling stage, we selected three cellulose synthase genes: Zm00001d032776 (CesA10), Zm00001d043477 (CesA11), and Zm00001d020531 (CesA12). The expression patterns of all six DEGs in the qRT-PCR assays were similar to those in the RNA-seq data, thus indicating the high reliability of the RNA-seq data.

\section{Discussion}

Maize stalks are composed of rind and pith. The rind is made up of hard and dense tissues, whereas the central pith of the stem is a much softer, foam-like tissue [29]. Previous research has noted the importance of rind thickness in maize stalk strength [2]. A recent study found that the cell walls of sclerenchyma cells of lodging-resistant lines were thicker than those of lodging-sensitive lines [30]. Similar results were observed in the present study. We found significant differences in rind thickness and cell wall thickness between the two SS lines and the NSS line (Fig. 2). Moreover, the two SS lines with stronger stalks 


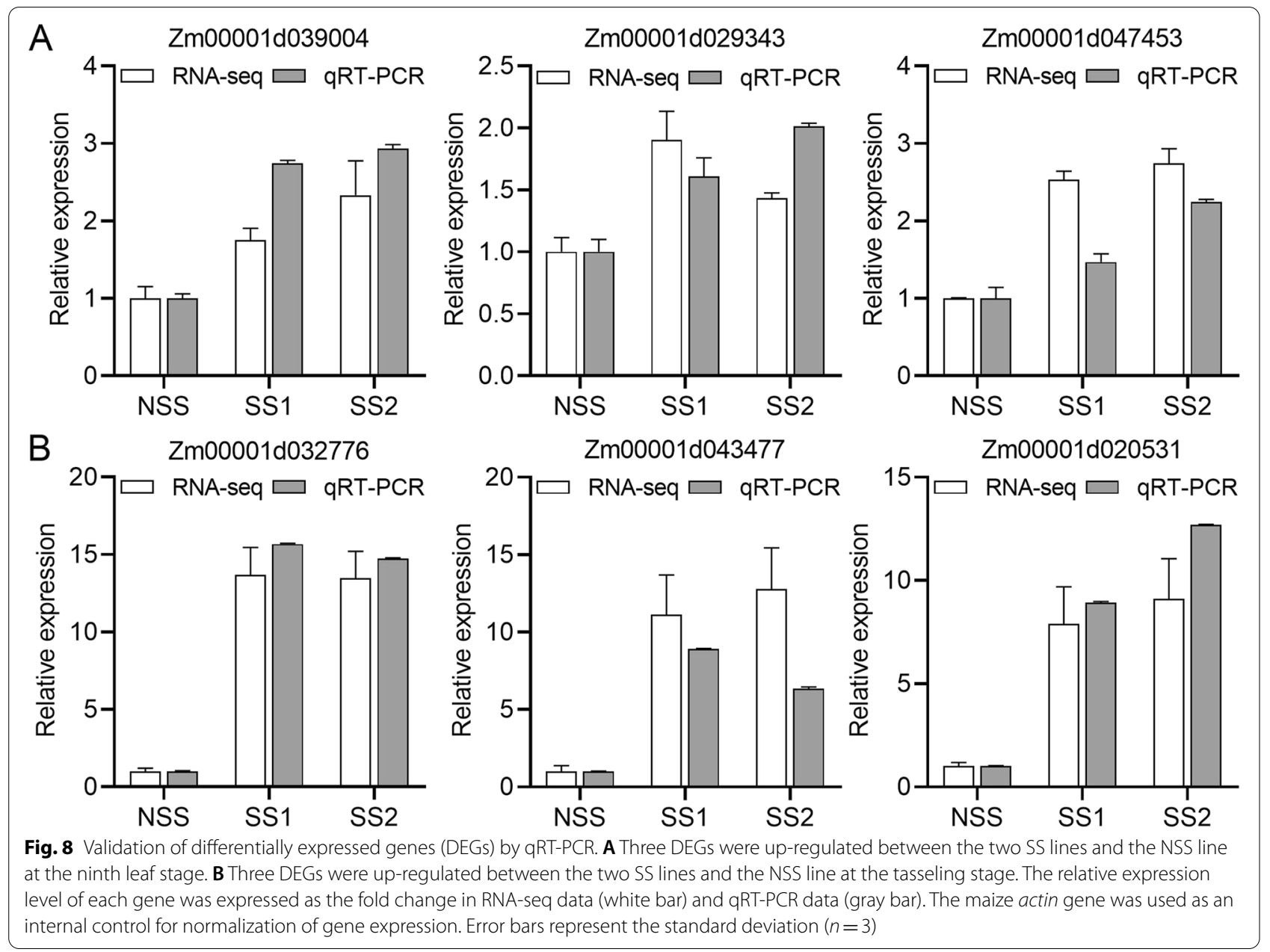

had more hemicellulose, cellulose, and lignin (Fig. 1D-F). According to these results, the structural polysaccharides and lignins of cell walls are related to the mechanical strength of the stalk, similar to the findings of previous studies [31]. Therefore, the thickness of the rind and cell wall might play important roles in maize stalk strength.

The plant cell wall is a complex matrix mainly consisting of polysaccharides, proteins, and lignins [20]. In particular, the changes in polysaccharides (cellulose, hemicellulose, and pectin) affect the cell wall mechanical strength and the viscoelastic properties of tissue [32]. Cell wall polysaccharides (hemicellulose and pectin) are synthesized by glycosyltransferases (GTs) in the Golgi apparatus and secreted into the cell wall by secretory vesicles [33]. Genes belonging to glycosyltransferase (GT) families were enriched in the transferase activity (transferring hexosyl) group (GO: 0016758, $p=6.46 \times 10^{-5}$ ) at the tasseling stage (Table S5). In the present study, a large number of UDP-glucosyl transferase genes $(33 / 85)$ were enriched in the transferase activity (transferring hexosyl) group (GO: 0016758, $p=6.46 \times 10^{-5}$ ) at the tasseling stage. UDP-glucosyl transferases mediate the transfer of glycosyl residues from activated nucleotide sugars (possibly UDP-glucose, UDP-galactose, UDP-glucuronic acid, or UDP-xylose) to acceptor molecules [34]. UDP glucose is a major substrate for the synthesis of cell wall polysaccharides, including cellulose, callose, xyloglucan, and glucomannan [35]. Xyloglucan and xylan are the two major hemicellulose polysaccharides in the cell walls of plants [14]. In Arabidopsis, the irregular xylem 9/-like (IRX9/ IRX9- $L$ ) and IRX14/IRX14- $L$ genes encode members of GT43, and the destruction of these proteins results in a decrease in xylan content [36]. In the present study, 12 GT43 genes, including the homologous genes of IRX9 (Zm00001d010976) and IRX14 (Zm00001d036543), were up-regulated in the two SS lines compared with the NSS line at the tasseling stage (Fig. 6 and Table S5). In maize, CesA10, CesA11 and CesA12 are cellulose synthases [37]. Compared with the NSS line, CesA10 (Zm00001d032776), CesA11 (Zm00001d043477) and CesA12 (Zm00001d020531) were all up-regulated in the two SS lines at the tasseling stage (Fig. 6 and Table S5). 
At the tasseling stage, maize inbred lines with different stalk strengths exhibited marked differences in cell wall synthesis. Compared with the NSS line, the two SS lines had higher cell wall polysaccharide contents (Fig. 1), which suggests that the two SS lines require more cell wall polysaccharides.

When cell wall polysaccharides produced in the Golgi apparatus are secreted into cell walls, multiple exoplasmic enzymes modify the structure of non-cellulosic polysaccharides, such as glycosyl hydrolases (GHs) [38, 39]. Xyloglucan endotransglucosylase/hydrolase (XTH), belonging to the GH16 family, plays a role in the plant cell wall structure and function [40]. XTH proteins have the activity of xyloglucan endotransglycosylase (XET) and xyloglucan endohydrolase, and act on xyloglucan attached to cellulose microfibers [41, 42]. In previous studies on XET activity in poplar, XET was found to play a role in secondary cell wall deposition [22]. In the present study, three XETs were up-regulated in two SS lines at the tasseling stage, which might affect hemicellulose deposition in the secondary cell wall (Fig. 6 and Table S5).

Mutation of the Arabidopsis COBRA-like 10 gene leads to the destruction of cellulose microfibril deposition in pollen tubes [23]. In rice, mutation of the COBRA-like protein gene brittle culm1 leads to a decrease in cellulose content and secondary cell wall thickness [24]. In the present study, six genes were predicted to encode COBRAlike proteins and were up-regulated in two SS lines at the tasseling stage (Fig. 6 and Table S7). Maize brittle stalk 2 encodes a COBRA-like protein similar to the rice brittle culm1 protein. Mutation in brittle stalk 2 affects cellulose deposition in the secondary cell wall and stalk strength $[25,43]$. Here, brittle stalk 2 (Zm00001d022081) was upregulated in the two SS lines at the tasseling stage (Fig. 6 and Table S7). Therefore, there might be a marked difference in cellulose deposition between the two SS lines and the NSS line.

Lignin, the second most abundant cell wall structural material after cellulose in the secondary cell wall, is very important for stem strength [44]. Previous studies found significantly reduced lignin content in Arabidopsis PAL1 and $P A L 2$ double mutants [16]. In the present study, five Arabidopsis PAL1 and PAL2 homologous genes, ZmPAL1 (Zm00001d017274), ZmPAL4 (Zm00001d051166), ZmPAL5 (Zm00001d051163), ZmPAL7 (Zm00001d017279), and ZmPAL8 (Zm00001d017276), were up-regulated in the two SS lines at the tasseling stage (Fig. 6 and Table S6). A recent study found that the Zm4CL1 mutation mainly affected the biosynthesis of $\mathrm{G}$ lignin and the accumulation of soluble feruloyl derivatives in maize lignified tissues [17]. Although there was no significant difference in $Z m 4 C L 1$ expression in our RNA-seq data, we found that another five $4 C L$ genes were up-regulated in the two SS lines at the tasseling stage (Fig. 6 and Table S6). Laccase is necessary for lignin polymerization during vascular development in Arabidopsis [18]. Our results showed that six laccase genes were up-regulated at the tasseling stage (Fig. 6 and Table S6). Under nitrogen stress, ZmmiR528 affected the lignin content and RPR of maize stems by regulating the expression of ZmLACCASE3 and ZmLACCASE5 [45]. Here, ZmLACCASE5 (Zm00001d042901) was up-regulated at the tasseling stage (Fig. 6 and Table S6). Taken together, enhanced expression of genes involved in cell wall synthesis and metabolism might increase cell wall structural material contents and cell wall thickness in the two SS lines, thereby improving stalk strength.

The cell wall structure is not only affected by the synthesis and modification of cell wall polysaccharides but is also regulated and organized by the dynamic cytoskeleton and multiple transport pathways of cell wall polymers [46]. The cytoskeleton is composed of microtubules and actin filaments (F-actin). Actin filament polymerization and elongation are related to vesicle mobility [47]. Microtubules are involved in the transport of vesicles containing cell wall components [48]. Cytoskeleton-associated proteins affect cell wall mechanics and cell wall material deposition $[13,49]$.

In this study, genes related to cytoskeleton organization (Table S3) and microtubule-based processes (Table S4) were enriched at the ninth leaf and tasseling stages, respectively. Actin-related protein 2/3 (Arp2/3) complex and $\mathrm{F}$-actin capping protein-related genes were up-regulated at the ninth leaf stage in the two SS lines compared with the NSS line. The Arp2/3 complex is a type of actin filament nucleation factor that extends a new actin filament with $70^{\circ}$ branch angles from one side of existing actin filaments [50]. The F-actin capping protein is a heterodimer composed of $\alpha$ and $\beta$ subunits that binds to the F-actin ends to limit the addition or loss of G-actin, thereby stabilizing the actin filament cytoskeleton [51]. Therefore, the Arp2/3 complex and F-actin capping proteins may affect the synthesis and nucleation of actin filaments and subsequently influence the transportation of cell wall polysaccharides during cell wall synthesis. In this study, capping protein subunits $\alpha$ (Zm00001d007146, $\log 2 \mathrm{FC}=0.44-0.54) \quad$ and $\beta \quad(Z m 00001 d 029343$, $\log 2 \mathrm{FC}=0.48-0.89$ ) were up-regulated in the two SS lines at the ninth leaf stage (Table S3). In addition, a large number of kinesin-related genes $(20 / 36)$ were enriched in the GO term of the microtubule-based process (GO: 0007017, $p=1.52 \times 10^{-5}$ ). Kinesin-1 is a processive motor that uses ATP energy to transport cellular cargoes from the Golgi apparatus to the cell periphery along the microtubules [52]. Kinesin-4-based transport of non-cellulosic substances along cortical microtubules is 
associated with cell wall mechanics in Arabidopsis thaliana [13]. The absence of the kinesin-4 family member FRAGILE FIBER1 (FRA1) kinesin results in the accumulation of vesicles around the Golgi apparatus and a reduction in pectin secretion [48]. A recent study found that FRA1 regulates the protein levels of cellulose synthasemicrotubules that uncouple protein and microtubule localization, which stabilizes the deposition sites of cellulose and cell wall polysaccharides [49]. In the present study, kinesin-related genes were differentially expressed at the tasseling stage (Table S4). These results suggest that kinesin might be involved in cell wall development through the transport of cell wall non-cellulosic material or influence the deposition of cellulose and cell wall polysaccharides.

Villin is an F-actin regulatory protein with a gelsolin-like core domain and a C-terminal villin headpiece (VHP) [53]. We found that VHP-related genes were differentially expressed between the two SS lines and the NSS line at the ninth leaf and tasseling stages. In the two combinations SS1vsNSS and SS2vsNSS, the expression level of Zm00001d047453 had the largest differences in cytoskeleton organization (GO: 0007010, $\left.p=1.00 \times 10^{-2}, \quad \log 2 \mathrm{FC}=1.32-1.41\right)$ and microtubule-based process (GO: 0007017, $p=1.52 \times 10^{-5}$, $\log 2 \mathrm{FC}=1.60-1.73)$. Further qRT-PCR assays also indicated that $Z m 00001 d 047453$ was differentially expressed between the two SS lines and the NSS line (Fig. 8A). Although the fold change of $Z m 00001 d 047453$ was lower in the qRT-PCR assay than indicated by the RNAseq data, the gene was up-regulated according to both datasets. Bao et al. [54] reported that the deletion of the VILLIN2 and VILLIN3 genes in Arabidopsis thaliana reduces stem mechanical strength. No defects in secondary cell wall synthesis were detected in that study; however, cortex, interfascicular fiber, xylem, and pith cell numbers significantly decreased, indicating defective sclerenchyma development. Therefore, we speculate that Zm00001d047453 affects maize stem differentiation and rind development.

\section{Conclusions}

At the tasseling and maturity stages, the two stiff-stalk lines had higher dry weights and hemicellulose, cellulose, and lignin contents per unit length than the non-stiffstalk line. With regard to microstructure, rind, VBSs, and rind and VBS cell walls were markedly thicker in the two stiff-stalk lines than in the non-stiff-stalk line at the tasseling stage. Transcriptome analysis revealed that these differences might be related to the expression levels of genes involved in cell wall metabolism and the cytoskeleton. Overall, our study has provided new insights into the internode development of maize inbred lines with different stalk strengths.

\section{Methods \\ Materials}

Maize inbred lines SJ20104 (NSS), HB08F1 (SS1), and A801 (SS2) were grown at the experimental station $\left(36^{\circ} 90^{\prime} \mathrm{N}, 117^{\circ} 90^{\prime} \mathrm{E}\right)$ of Shandong Agricultural University. The soil type was brown soil with a sandy loam structure containing $10.5 \mathrm{~g} \mathrm{~kg}^{-1}$ organic matter, $0.8 \mathrm{~g} \mathrm{~kg}^{-1}$ total nitrogen $(\mathrm{N}), 35.2 \mathrm{mg} \mathrm{kg}^{-1}$ readily available phosphorus $(\mathrm{P})$, and $81.8 \mathrm{mg} \mathrm{kg}^{-1}$ readily available potassium (K). N, $\mathrm{P}$, and $\mathrm{K}$ fertilizers were applied as described previously [55]. Seeds were sown on 12 June 2019. Each field plot was $4 \mathrm{~m} \times 4.8 \mathrm{~m}$ with eight rows $(0.60 \mathrm{~m}$ between rows, with a planting density of 67,500 plants $\mathrm{ha}^{-1}$ ).

\section{Plant height, internode morphology, and RPR measurements}

At the maturity stage, plant height was measured from the ground to the top of the tassel. Stem diameter was determined with a vernier caliper arranged perpendicular to the long axis (Fig. 1A) at the center of the third basal internode. The length of the third basal internode was measured with a ruler.

Five individual plants were randomly selected from each maize inbred line at ninth leaf, tasseling, and maturity stages. Days from sowing to ninth leaf, tasseling, and maturity stages were 36, 50, and 102 in NSS; 36, 51, and 104 in SS1; and 38, 59, and 108 in SS2, respectively. NSS, SS1, and SS2 had a total of 16, 16, and 18 leaves at the tasseling stage, respectively. The RPR of the third basal internode was measured with a YYD-1 stalk strength tester (Top Cloud-Agri Technology Co., Zhejiang, China) according to a previous study [56]. In brief, a 1-cm-long probe with a $1-\mathrm{mm}^{2}$ cross-sectional area was inserted perpendicular to the long axis of the cross section at the center point of the third basal internode (Fig. 1A), and the maximum value was recorded as the RPR. Then, the middle part of the third basal internode was cut approximately $2 \mathrm{~mm}$ in length (Fig. 1A). The cut part was cut in half along the long axis of the cross-section. One half, designated sample 1, was used for microstructure observation. The other half (as sample 2) was immediately frozen in liquid nitrogen and stored at $-80^{\circ} \mathrm{C}$ for RNA extraction.

\section{Determination of hemicellulose, cellulose, and lignin contents}

Another five plants were randomly selected from each maize inbred line for determination of hemicellulose, cellulose, and lignin contents. The entire third basal internode was cut off, heated in an air oven at $105^{\circ} \mathrm{C}$ 
for $30 \mathrm{~min}$, and then dried to a constant weight at $80^{\circ} \mathrm{C}$. The DWUL of the third internode was calculated by dividing the dry weight of the internode by its length. The dried sample was then crushed and passed through a 40-mesh screen. Approximately $1 \mathrm{~g}$ of the sifted sample (M1) was placed in a filter bag (with a weight of M0) for measurement of neutral detergent fiber (NDF), acid detergent fiber (ADF), and acid detergent lignin (ADL) contents using an ANKOM 220 fiber analyzer (ANKOM Technology Corp., Fairport, NY, USA) according to a previous study [55]. Hemicellulose, cellulose, and lignin percentages were calculated as follows: hemicellulose $(\%)=(\mathrm{NDF}-\mathrm{ADF}) / \mathrm{M} 1$; cellulose $(\%)=(\mathrm{ADF}-\mathrm{ADL}) / \mathrm{M} 1$; and lignin $(\%)=(\mathrm{ADL}-\mathrm{M} 0) /$ M1. The percentage of each compound was multiplied by DWUL to obtain hemicellulose (HWUL), cellulose (CWUL), and lignin (LWUL) weights per unit length of the third basal internode $\left(\mathrm{mg} \mathrm{cm}^{-1}\right)$. Each component was measured three times per sample.

\section{Paraffin sectioning and histological staining}

Samples for microstructure observation were obtained from the middle part of the third basal internode as described above for sample 1. Tissues at the ninth leaf and tasseling stages were immersed in 50 and $70 \%$ formalin-acetic acid-alcohol (FAA) fixative, respectively. Paraffin sectioning procedures were performed as previously described [57]. Samples were dehydrated in a graded ethanol series, soaked in 50\% pure ethanol:50\% xylene (v/v) for $45 \mathrm{~min}$ and $100 \%(\mathrm{v} / \mathrm{v})$ xylene for $45 \mathrm{~min}$ (repeated three times), and then infiltrated with liquefied paraffin at $58^{\circ} \mathrm{C}$. Paraffin sections were produced with an HM360 slicer (Microm, Walldorf, Germany). The sections were dewaxed with xylene for $20 \mathrm{~min}$ (repeated twice), anhydrous ethanol for $5 \mathrm{~min}$ (repeated twice), and 75\% alcohol for $5 \mathrm{~min}$, and rinsed with water. The sections were dyed in Safranin-O for $2 \mathrm{~h}$, and washed in water to remove excess dye. Then, the slices were placed in 50, 70 and $80 \%$ gradient alcohol for $3-8 \mathrm{~s}$ for decolorization. The slices were dyed in Fast Green solution for 6-20s and dehydrated with absolute ethanol (repeated three times). Xylene was transparent for $5 \mathrm{~min}$ and sealed with neutral gum. Sections were imaged by Nikon DS-Ri2 light microscopy (Nikon, Tokyo, Japan). Measurements of the stem rind, VBS, and cell wall thicknesses were carried out using ImageJ software. Rind thickness was measured 10 times randomly from multiple directions in the cross section of each inbred line $(5 \times$ magnification). VBS thickness was measured from the thickest part of 10 randomly selected VBSs of each inbred line $(10 \times$ magnification, shown as a red line in Fig. 2A). Rind cell wall thickness was measured from thick-walled cells in the center of the rind (except for the vascular bundle). VBS cell wall thickness was measured from randomly selected VBSs. Two adjacent cells were measured simultaneously, and 20 pairs of cells were measured (40× magnification). Cell wall thickness was then calculated by dividing each measured value in half.

\section{RNA-seq analysis}

Samples of SS1, SS2, and NSS at the ninth leaf and tasseling stages were used for RNA-seq analysis. These samples for RNA extraction were obtained from the middle part of the third basal internode as described above (as sample 2). Each frozen sample was ground in liquid nitrogen in a $50 \mathrm{~mL}$ stainless steel grinding jar with one $20 \mathrm{~mm}$ stainless steel ball (AM100, Ant Source Scientific Instrument Co., Beijing, China). RNA isolation and quantification were carried out as described previously [58]. In brief, total RNA was extracted from $0.1 \mathrm{~g}$ of sample using an RNA extraction kit DP441 (Tiangen, Beijing, China). RNA degradation and contamination were detected by $1 \%$ agarose gel electrophoresis, and a NanoPhotometer spectrophotometer (Implen, CA, USA) was used to determine RNA purity. RNA integrity and concentration were assessed using an Agilent 2100 bioanalyzer (Agilent Technologies, CA, USA) and a Qubit RNA assay kit with a Qubit 2.0 fluorometer (Life Technologies, CA, USA), respectively. RNA integrity numbers (RIN) ranged from 7.9 to 9.8 (Table S9), thus indicating that the RNA quality was good.

RNA-seq library construction and sequencing were carried out at Beijing Novogene Bioinformatics Technology Co. (Beijing, China). A NEBNext UltraTM RNA Library Prep Kit for Illumina (NEB, MA, USA) was used for RNA-seq library construction. By using a poly$\mathrm{T}$ oligo-attached magnetic, mRNA was purified from total RNA. First-strand cDNA synthesis was carried out with random hexamer primers and M-MuLV reverse transcriptase (RNase $\mathrm{H}-$ ). Then, RNase $\mathrm{H}$ and DNA polymerase I were used to synthesize second strand cDNA. After adenylation, the AMPure XP system (Beckman Coulter, MA, USA) was used to purify cDNA fragments $(250 \sim 300 \mathrm{bp})$. The libraries were sequenced on an Illumina NovaSeq platform to generate $150 \mathrm{bp}$ paired-end reads. Clean data were obtained by removing adapter-containing reads, reads containing poly- $\mathrm{N}$, and low-quality reads from the raw data. Subsequently, the clean reads were mapped to the maize B73 $\mathrm{v} 4$ reference genome (ftp://ftp.ensemblgenomes.org/pub/ plants/release-41/fasta/zea_mays/dna/) using HISAT2 [59]. To quantify the gene expression levels, the number of reads mapped to each gene were counted using featureCounts [60], and the expected number of FPKM of each gene were calculated. Expressed genes with 
FPKM $\geq 1$ were used for the comparative analysis [61]. Differential expression analysis of two samples was performed using the DESeq2 R package (v1.16.1) [62]. The $p$-value were adjusted using the Benjamini and Hochberg algorithm to control the false discovery rate [63], and genes with corrected $p$-value $<0.05$ were considered to be DEGs. To understand the functions of significant DEGs, we applied the clusterProfiler R package [64] to enable a GO enrichment analysis. GO terms with a corrected $p$-value $<0.05$ were considered to be significantly enriched in DEGs.

\section{qRT-PCR}

Gene-specific primers designed using Primer 6 software were synthesized by Sangon Biotech (Shanghai, China). The primer sequences are listed in Table S10. cDNA was reverse transcribed from total RNA using a PrimeScript RT kit (Takara, Dalian, China) and then analyzed by qRT-PCR on an ABI StepOne Plus Real-Time PCR system (Applied Biosystems, CA, USA). The maize actin gene (Zm00001d010159) was used as an internal control for normalization of gene expression. The actin primer sequences were obtained from a previous study [65]. Each qRT-PCR experiment was repeated three times. Relative expression levels were calculated using the comparative CT $\left(2^{-\Delta \Delta \mathrm{Ct}}\right)$ method.

\section{Statistical analyses}

One-way ANOVA and Duncan's post hoc test were conducted using SPSS 19.0 statistical software (SPSS, Inc., IL, USA).

\begin{abstract}
Abbreviations
NSS: Non-stiff-stalk; SS: Stiff-stalk; RPR: Rind penetrometer resistance; CesA: Cellulose synthase; CSCs: Cellulose synthase complexes; DWUL: Dry weight per unit length; HWUL: Hemicellulose weight per unit length; CWUL: Cellulose weight per unit length; LWUL: Lignin weight per unit length; VBS: Vascular bundle sheath; FPKM: Fragments per kilobase of transcript sequence per million base pairs sequenced; DEGs: Differentially expressed genes; GO: Gene Ontology; XET: Xyloglucan endo-transglycosylase; GH: Glycosyl hydrolase; GT: Glycosyltransferase; XTH: Xyloglucan endotransglucosylase/hydrolase; PAL: Phenylalnine ammonialyase; $4 \mathrm{CL}$ : 4-coumarate:CoA ligase; TF: Transcription factor; XET: Xyloglucan endotransglycosylase; IRX: Irregular xylem; Arp2/3: Actin-related protein 2/3; FRA1: FRAGILE FIBER1; VHP: Villin headpiece; NDF: Neutral detergent fiber; ADF: Acid detergent fiber; ADL: Acid detergent lignin.
\end{abstract}

\section{Supplementary Information}

The online version contains supplementary material available at https://doi. org/10.1186/s12870-022-03435-w.

Additional file 1: Fig. S1. Comparison of stem diameters, internode lengths, and plant height.

Additional file 2: Fig. S2. Venn diagram of down-regulated DEGs in SS1vsNSS and SS2vsNSS.
Additional file 3: Fig. S3. Top 30 significantly enriched Gene Ontology $(\mathrm{GO})$ terms $(p<0.05)$ in DEGs commonly down-regulated in SS1vsNSS and SS2VsNSS at the ninth leaf stage.

Additional file 4: Fig. S4. Top 30 significantly enriched Gene Ontology $(G O)$ terms $(p<0.05)$ in DEGs commonly down-regulated in SS1vsNSS and SS2vsNSS at the tasseling stage.

Additional file 5: Table S1. Summary of RNA-seq data.

Additional file 6: Table S2. List of selected genes related to hydrolase activity (hydrolyzing O-glycosyl compounds) that were up-regulated in SS1VsNSS and SS2VsNSS at the ninth leaf stage.

Additional file 7: Table S3. List of selected cytoskeleton organizationrelated genes that were up-regulated in SS1VSNSS and SS2vsNSS at the ninth leaf stage.

Additional file 8: Table S4. List of selected microtubule-based processrelated genes that were up-regulated in SS1vsNSS and SS2VsNSS at the tasseling stage.

Additional file 9: Table S5. List of selected genes related to transferase activity (transferring hexosyl groups) that were up-regulated in SSIvsNSS and SS2vsNSS at the tasseling stage.

Additional file 10: Table S6. Lignin synthesis related genes that were upregulated in SS1vsNSS and SS2VSNSS at the tasseling stage.

Additional file 11: Table S7. List of selected plant-type cell wall organization-related genes that were up-regulated in SSIVSNSS and SS2vsNSS at the tasseling stage.

Additional file 12: Table S8. Differentially expressed transcription factors in SS1vsNSS and SS2VsNSS.

Additional file 13: Table S9. RNA integrity number (RIN) values.

Additional file 14: Table S10. Primer sequences for qRT-PCR analysis.

\section{Acknowledgements}

We are grateful for the funding support.

\section{Authors' contributions}

X.L., D.W., and C.Z. conceived of the study. X.L. and W.C. performed most of the experiments. X.L. analyzed the results. X.L., D.W., and C.Z. wrote the manuscript. All authors read and approved the final manuscript.

\section{Funding}

This work was supported by the Shandong Improved Variety Program (2019LZGC002-1) and the Modern Agricultural Industry Technology System Innovation Team of Shandong Province of China (SDAIT-02-02).

\section{Availability of data and materials}

The original contributions presented in the study are publicly available. The RNA-seq data have been deposited in the NCBI Sequence Read Archive, accession number: PRJNA752936 (https://www.ncbi.nlm.nih.gov/sra/PRJNA 752936).

\section{Declarations}

Ethics approval and consent to participate Not applicable.

\section{Consent for publication}

Not applicable.

\section{Competing interests}

The authors declare that the research was conducted in the absence of any commercial or financial relationships that could be construed as a potential conflict of interest.

Received: 10 October 2021 Accepted: 11 January 2022

Published online: 24 January 2022 


\section{References}

1. Kamran M, Cui W, Ahmad I, Meng X, Zhang X, Su W, et al. Effect of paclobutrazol, a potential growth regulator on stalk mechanical strength, lignin accumulation and its relation with lodging resistance of maize. Plant Growth Regul. 2018;84(2):317-32.

2. Robertson DJ, Julias M, Lee SY, Cook DD. Maize stalk lodging: morphological determinants of stalk strength. Crop Sci. 2017;57(2):926-34.

3. Heckwolf S, Heckwolf M, Kaeppler SM, De Leon N, Spalding EP. Image analysis of anatomical traits in stalk transections of maize and other grasses. Plant Methods. 2015;11:26.

4. Cosgrove DJ, Jarvis MC. Comparative structure and biomechanics of plant primary and secondary cell walls. Front Plant Sci. 2012;3:204.

5. Cook DD, Meehan K, Asatiani L, Robertson DJ. The effect of probe geometry on rind puncture resistance testing of maize stalks. Plant Methods. 2020;16:65

6. Cosgrove DJ. Growth of the plant cell wall. Nat Rev Mol Cell Biol. 2005;6(11):850-61.

7. Brown RM, Saxena I, Kudlicka K. Cellulose biosynthesis in higher plants. Trends Plant Sci. 1996;1(5):149-56.

8. Mohnen D. Pectin structure and biosynthesis. Curr Opin Plant Biol. 2008;11(3):266-77.

9. Scheller HV, Ulvskov P. Hemicelluloses. Annu Rev Plant Biol. 2010;61:263-89.

10. Watanabe Y, Meents MJ, McDonnell LM, Barkwill S, Sampathkumar A, Cartwright HN, et al. Visualization of cellulose synthases in Arabidopsis secondary cell walls. Science. 2015;350(6257):198-203.

11. Driouich A, Faye L, Staehelin A. The plant Golgi apparatus: a factory for complex polysaccharides and glycoproteins. Trends Biochem Sci. 1993;18(6):210-4

12. Parrotta L, Faleri C, Cresti M, Cai G. Heat stress affects the cytoskeleton and the delivery of sucrose synthase in tobacco pollen tubes. Planta. 2016;243(1):43-63.

13. Kong Z, loki M, Braybrook S, Li S, Ye ZH, Julie Lee YR, et al. Kinesin-4 functions in vesicular transport on cortical microtubules and regulates cell wall mechanics during cell elongation in plants. Mol Plant. 2015:8(7):1011-23.

14. Zhong R, Teng Q, Haghighat M, Yuan Y, Furey ST, Dasher RL, et al. Cytosol-localized UDP-xylose synthases provide the major source of UDPxylose for the biosynthesis of xylan and xyloglucan. Plant Cell Physiol. 2017;58(1):156-74.

15. Lee C, Teng Q, Zhong R, Yuan Y, Ye Z-H. Functional roles of rice glycosyltransferase family GT43 in xylan biosynthesis. Plant Signal Behav. 2014;9:e27809.

16. Rohde A, Morreel K, Ralph J, Goeminne G, Hostyn V, De Rycke R, et al. Molecular phenotyping of the pal1 and pal 2 mutants of Arabidopsis thaliana reveals far-reaching consequences on phenylpropanoid, amino acid, and carbohydrate metabolism. Plant Cell. 2004;16(10):2749-71.

17. Xiong W, Wu Z, Liu Y, Li Y, Su K, Bai Z, et al. Mutation of 4-coumarate: coenzyme a ligase 1 gene affects lignin biosynthesis and increases the cell wall digestibility in maize brown midrib5 mutants. Biotechnol Biofuels. 2019;12:82.

18. Zhao Q, Nakashima J, Chen F, Yin Y, Fu C, Yun J, et al. LACCASE is necessary and nonredundant with PEROXIDASE for lignin polymerization during vascular development in Arabidopsis. Plant Cell. 2013;25(10):3976-87.

19. Morrison TA, Jung HG, Buxton DR, Hatfield RD. Cell-wall composition of maize internodes of varying maturity. Crop Sci. 1998;38(2):455-60.

20. Zhang B, Gao Y, Zhang L, Zhou Y. The plant cell wall: biosynthesis, construction, and functions. J Integr Plant Biol. 2021;63(1):251-72.

21. Sharples SC, Nguyen-Phan TC, Fry SC. Xyloglucan endotransglucosylase/ hydrolases (XTHs) are inactivated by binding to glass and cellulosic surfaces, and released in active form by a heat-stable polymer from cauliflower florets. J Plant Physiol. 2017:218:135-43.

22. Bourquin V, Nishikubo N, Abe H, Brumer H, Denman S, Eklund M, et al. Xyloglucan endotransglycosylases have a function during the formation of secondary cell walls of vascular tissues. Plant Cell. 2002;14(12):3073-88.

23. Li S, Ge FR, Xu M, Zhao XY, Huang GQ, Zhou LZ, et al. Arabidopsis COBRALIKE 10, a GPI-anchored protein, mediates directional growth of pollen tubes. Plant J. 2013;74(3):486-97.

24. Li Y, Qian Q, Zhou Y, Yan M, Sun L, Zhang M, et al. BRITTLE CULM1, which encodes a COBRA-like protein, affects the mechanical properties of rice plants. Plant Cell. 2003;15(9):2020-31.
25. Sindhu A, Langewisch T, Olek A, Multani DS, McCann MC, Vermerris W et al. Maize Brittle stalk2 encodes a COBRA-like protein expressed in early organ development but required for tissue flexibility at maturity. Plant Physiol. 2007;145(4):1444-59.

26. Wessels B, Seyfferth C, Escamez S, Vain T, Antos K, Vahala J, et al. An AP2 ERF transcription factor ERF 139 coordinates xylem cell expansion and secondary cell wall deposition. New Phytol. 2019:224(4):1585-99.

27. Taylor-Teeples M, Lin L, De Lucas M, Turco G, Toal TW, Gaudinier A, et al. An Arabidopsis gene regulatory network for secondary cell wall synthesis. Nature. 2015;517(7536):571-5.

28. Ye Y, Wu K, Chen J, Liu Q, Wu Y, Liu B, et al. OsSND2, a NAC family transcription factor, is involved in secondary cell wall biosynthesis through regulating MYBs expression in rice. Rice. 2018;11(1):36.

29. Stubbs CJ, Sun W, Cook DD. Measuring the transverse young's modulus of maize rind and pith tissues. J Biomech. 2019:84:113-20.

30. Guo Y, Hu Y, Chen $H$, Yan P, Du Q, Wang Y, et al. Identification of traits and genes associated with lodging resistance in maize. Crop J. 2021:9(6):1408-17.

31. Martin SA, Darrah LL, Hibbard BE. Divergent selection for rind penetrometer resistance and its effects on european corn borer damage and stalk traits in corn. Crop Sci. 2004;44(3):711-7.

32. Videcoq P, Barbacci A, Assor C, Magnenet V, Arnould O, Le Gall S, et al. Examining the contribution of cell wall polysaccharides to the mechanical properties of apple parenchyma tissue using exogenous enzymes. J Exp Bot. 2017;68(18):5137-46.

33. Hoffmann N, King S, Samuels AL, McFarlane HE. Subcellular coordination of plant cell wall synthesis. Dev Cell. 2021;56(7):933-48.

34. Huang FF, Chai CL, Zhang Z, Liu ZH, Dai FY, Lu C, et al. The UDP-glucosyltransferase multigene family in Bombyx mori. BMC Genomics. 2008:9:563.

35. Bar-Peled M, O'Neill MA. Plant nucleotide sugar formation, interconversion, and salvage by sugar recycling. Annu Rev Plant Biol. 2011:62:127-55.

36. Wu AM, Hörnblad E, Voxeur A, Gerber L, Rihouey C, Lerouge P, et al. Analysis of the Arabidopsis IRX9/IRX9- $L$ and IRX14/IRX14- $L$ pairs of glycosyltransferase genes reveals critical contributions to biosynthesis of the hemicellulose glucuronoxylan. Plant Physiol. 2010;153(2):542-54.

37. Appenzeller L, Doblin M, Barreiro R, Wang H, Niu X, Kollipara K, et al. Cellulose synthesis in maize: isolation and expression analysis of the cellulose synthase (CesA) gene family. Cellulose. 2004;11(3/4):287-99.

38. McFarlane HE, Watanabe Y, Gendre D, Carruthers K, Levesque-Tremblay G, Haughn GW, et al. Cell wall polysaccharides are mislocalized to the vacuole in echidna mutants. Plant Cell Physiol. 2013;54(11):1867-80.

39. Voiniciuc C, Pauly M, Usadel B. Monitoring polysaccharide dynamics in the plant cell wall. Plant Physiol. 2018;176(4):2590-600.

40. Jiang Y, Li Y, Lu C, Tang Y, Jiang X, Gai Y. Isolation and characterization of Populus xyloglucan endotransglycosylase/hydrolase (XTH) involved in osmotic stress responses. Int J Biol Macromol. 2020;155:1277-87.

41. Vissenberg K, Fry SC, Pauly M, Höfte H, Verbelen JP. XTH acts at the microfibril-matrix interface during cell elongation. J Exp Bot. 2005:56(412):673-83.

42. Rose JKC, Braam J, Fry SC, Nishitani K. The XTH family of enzymes involved in xyloglucan endotransglucosylation and endohydrolysis: current perspectives and a new unifying nomenclature. Plant Cell Physiol. 2002:43(12):1421-35

43. Ching A, Dhugga KS, Appenzeller L, Meeley R, Bourett TM, Howard RJ, et al. Brittle stalk 2 encodes a putative glycosylphosphatidylinositolanchored protein that affects mechanical strength of maize tissues by altering the composition and structure of secondary cell walls. Planta. 2006:224(5):1174-84

44. Boerjan W, Ralph J, Baucher M. Lignin biosynthesis. Annu Rev Plant Biol. 2003:54(1):519-46.

45. Sun Q, Liu X, Yang J, Liu W, Du Q, Wang H, et al. MicroRNA528 affects lodging resistance of maize by regulating lignin biosynthesis under nitrogenluxury conditions. Mol Plant. 2018;11(6):806-14.

46. Bashline L, Lei L, Li S, Gu Y. Cell wall, cytoskeleton, and cell expansion in higher plants. Mol Plant. 2014;7(4):586-600.

47. Liu C, Zhang Y, Ren H. Actin polymerization mediated by AtFH5 directs the polarity establishment and vesicle trafficking for pollen germination in Arabidopsis. Mol Plant. 2018;11(11):1389-99.

48. Zhu C, Ganguly A, Baskin TI, McClosky DD, Anderson CT, Foster C, et al. The fragile Fiber1 kinesin contributes to cortical microtubule-mediated trafficking of cell wall components. Plant Physiol. 2015;167(3):780-92. 
49. Ganguly A, Zhu C, Chen W, Dixit R. FRA1 kinesin modulates the lateral stability of cortical microtubules through cellulose synthase-microtubule uncoupling proteins. Plant Cell. 2020;32(8):2508-24.

50. Firat-Karalar EN, Welch MD. New mechanisms and functions of actin nucleation. Curr Opin Cell Biol. 2011;23(1):4-13.

51. Wang J, Qian D, Fan T, Jia H, An L, Xiang Y. Arabidopsis actin capping protein (AtCP) subunits have different expression patterns, and downregulation of AtCPB confers increased thermotolerance of Arabidopsis after heat shock stress. Plant Sci. 2012;193-194:110-9.

52. Angerani S, Lindberg E, Klena N, Bleck CKE, Aumeier C, Winssinger N. Kinesin-1 activity recorded in living cells with a precipitating dye. Nat Commun. 2021;12(1):1463.

53. Miears HL, Gruber DR, Horvath NM, Antos JM, Young J, Sigurjonsson JP, et al. Plant villin headpiece domain demonstrates a novel surface charge pattern and high affinity for F-actin. Biochemistry. 2018:57(11):1690-701.

54. Bao C, Wang J, Zhang R, Zhang B, Zhang H, Zhou Y, et al. Arabidopsis VILLIN2 and VILLIN3 act redundantly in sclerenchyma development via bundling of actin filaments. Plant J. 2012;71(6):962-75.

55. Zhao L, Xie L, Huang J, Su Y, Zhang C. Proper glyphosate application at post-anthesis lowers grain moisture content at harvest and reallocates non-structural carbohydrates in maize. Front Plant Sci. 2020;11:580883.

56. Wang X, Zhang R, Shi Z, Zhang Y, Sun X, Ji Y, et al. Multi-omics analysis of the development and fracture resistance for maize internode. Sci Rep. 2019;9(1):1.

57. Cai S, Lashbrook CC. Laser capture microdissection of plant cells from tape-transferred paraffin sections promotes recovery of structurally intact RNA for global gene profiling. Plant J. 2006;48(4):628-37.

58. Wen $\mathrm{D}, \mathrm{Xu} H$, Xie L, He M, Hou H, Zhang C. A loose endosperm structure of wheat seed produced under low nitrogen level promotes early germination by accelerating water uptake. Sci Rep. 2017;7(1):3116.

59. Kim D, Langmead B, Salzberg SL. HISAT: a fast spliced aligner with low memory requirements. Nat Methods. 2015;12(4):357-60.

60. Liao Y, Smyth GK, Shi W. featureCounts: an efficient general purpose program for assigning sequence reads to genomic features. Bioinformatics. 2014;30(7):923-30.

61. Ning L, Lin Z, Gu J, Gan L, Li Y, Wang H, et al. The initial deficiency of protein processing and flavonoids biosynthesis were the main mechanisms for the male sterility induced by SX-1 in Brassica napus. BMC Genomics. 2018;19(1):806.

62. Love MI, Huber W, Anders S. Moderated estimation of fold change and dispersion for RNA-seq data with DESeq2. Genome Biol. 2014;15(12):550.

63. Benjamini Y, Hochberg Y. Controlling the false discovery rate: a practical and powerful approach to multiple testing. J R Stat Soc B. 1995;57(1):289-300

64. Yu G, Wang LG, Han Y, He QY. clusterProfiler: an R package for comparing biological themes among gene clusters. OMICS. 2012;16(5):284-7.

65. Zhang Z, Yang J, Wu Y. Transcriptional regulation of zein gene expression in maize through the additive and synergistic action of opaque2, prolamine-box binding factor, and $\mathrm{O} 2$ heterodimerizing proteins. Plant Cell. 2015;27(4):1162-72.

\section{Publisher's Note}

Springer Nature remains neutral with regard to jurisdictional claims in published maps and institutional affiliations.

Ready to submit your research? Choose BMC and benefit from:

- fast, convenient online submission

- thorough peer review by experienced researchers in your field

- rapid publication on acceptance

- support for research data, including large and complex data types

- gold Open Access which fosters wider collaboration and increased citations

- maximum visibility for your research: over $100 \mathrm{M}$ website views per year

At BMC, research is always in progress.

Learn more biomedcentral.com/submissions 